\title{
Electro-physical properties of $\gamma$-exposed crystals of silicon and germanium
}

\author{
Yu. P. Dotsenko \\ Institute of Semiconductor Physics, National Academy of Sciences of Ukraine, Kyiv, 252028, Ukraine
}

\begin{abstract}
The paper represents a review of research data upon changing electrophysical properties of $\mathrm{n}-\mathrm{Si}$ and $\mathrm{n}-\mathrm{Ge}$ when radiation defects arise under action of different $\gamma$-irradiation doses. Analyzed are consequences of arising deep levels of radiation defects in the forbidden band of silicon and germanium, which leads to the considerable increase of resistivity gradients caused by non-uniform compensation of shallow donor centers. In addition, considered are characteristics of radiation defects energy levels which determine both regularities of electrophysical properties changes and peculiarities of tensoeffects in $\gamma$-irradiated crystals. It is noticed that neutron-doped $n$-Si $(\mathrm{P})$ has larger radiation hardness in respect to $\gamma$-irradiation as sompared to silicon doped with phosphorus in the course of crystal growth.
\end{abstract}

Keywords: radiation defects, $\gamma$-irradiation, electron silicon, electron germanium, radiation hardness.

Paper received 10.02.99; revised manuscript received 17.05.99; accepted for publication 24.05.99.

\section{Introduction}

Because the semiconductor exposure by high energy particles can be used with the objective to necessary change their properties, the investigations of the influence of different $\gamma$-exposure doses on the defect structure and characteristics of materials have not only scientific interest, but are also important and from the practical point of view. Besides that, due to the exposure action the change of the properties of the semiconductor materials, structures and devices, which operate in the radiation fields take place. Thus, from the point of view of prediction of the parameter stability of such devices the investigations of the regularities of creation and transformation of radiation defects in semiconductors under the radiation exposure is becoming more and more actual.

The investigations by different methods have established [1-11], that at the growth of bulk semiconductor crystals in the planes, perpendicular to the growth direction, the practically periodical distribution of the doping impurity arises. Depending on the crystal growth condi- tions [2] the periodicity of such structures is characterized by the width in the range of $10^{-6}-10^{-3} \mathrm{~m}$ and is connected with the different impurity distribution coefficient between liquid and solid phases of crystal, which is grown from the melt. The impurity concentration in the layers can differ by several times [12], because due to the charge carriers diffusion in the crystal volume, the intrinsic electrical field arises, which is modulated in the direction of the growth axis with the same periodicity as that of the impurity distribution [5]. A theoretical approach to the methods of taking into account in the kinetic phenomena the contribution of statistically distributed impurities in the crystal bulk was proposed by Herring [8]. It was shown, in particular, that transverse magnetic resistance connected with the growth layers, in classically strong fields is determined by the dependence $\Delta \rho_{\perp} / \rho_{0} \sim H^{2}$, and in the region of quantizing magnetic fields: $\Delta \rho_{\perp} / \rho_{0} \sim$ $\sim H$. The experimental investigations have confirmed the theoretical conclusions [ 9, 13, 14]. The corresponding 
Yu. P. Dotsenko al.: Electro-physical properties of $\boldsymbol{\gamma}$-exposed crystals ...

choice of orientation for current $\mathbf{J}$ and magnetic field $\mathbf{H}$ vector enabled to determine the transverse magnetic resistance of $n$-Ge which is directly connected with the presence of the defect structure in the form of growth layers.

Because as a result of $\gamma$-exposure in the crystal volume the defects are created which have the acceptor levels in the forbidden gap and which cause the compensation of the donor impurity, the properties of semiconductors with the increase of radiation dose are sufficiently changed, in particular, due to the essential growth of the gradients of the crystal specific resistance, which are determined by the presence of the growth layers [15-18].

It was proved that the most informative methods for the investigations of the origin and properties of the radiation defects are the combinations of different experimental methods (electron paramagnetic resonance (EPR), capacitance spectroscopy (DLTS), nuclear magnetic resonance (NMR), optical methods, etc.) with the high pressure methods, in particular, strong directed stresses. Therefore, considerable attention was paid to the investigation of the electro-physical and, in particular, tensor resistive properties of the $\gamma$-exposed semiconductors [15-21].

At present work the review of the literature and original data concerning the investigations of the electrophysical properties of the strongly deformed $\gamma$-exposed crystals of silicon and germanium of n-type will be adduced.

\section{Influence of the $\gamma$-radiation on the electro- physical properties of $n$-type silicon}

Because the most informative from the stand point of studying the influence of $\gamma$-radiation on the properties of materials are the investigation results at different exposure doses, including those ones which lead to the drastic changes of the semiconductor properties (for example, strong compensation, $n-p$ conversion, essential increase of tensor sensitivity, etc.) the investigation results of the influence of different $\gamma$-exposure doses on electro-physical and, in particular, tensoresistive properties of silicon and germanium will be presented in present review.

The results of the investigations of the $\gamma$-exposure influence on the tensor resistive properties of silicon, doped by the phosphor during growth process $[22,23]$, are presented in Fig. 1. Let us notice that the silicon crystals which were grown in the [100] direction, were doped by the phosphor impurity from the melt up to concentrations $1.2 \times 10^{14} \mathrm{~cm}^{-3}$. It can be seen from the presented in Fig. 1 data, that after the certain $\gamma$-exposure doses $\Phi \approx$ $\approx 1.7 \times 10^{17}$ quanta $/ \mathrm{cm}^{2}$ ) the essential anisotropy of $\rho_{X} / \rho_{0}=f(X)$ dependencies for the samples fabricated in equivalent crystallographic directions ([100] - samples of I group and [001] - samples of II group [22, 23]), but with different orientation relative to the direction of the crystal growth (curves 3-3', 4-4') arises. The type of

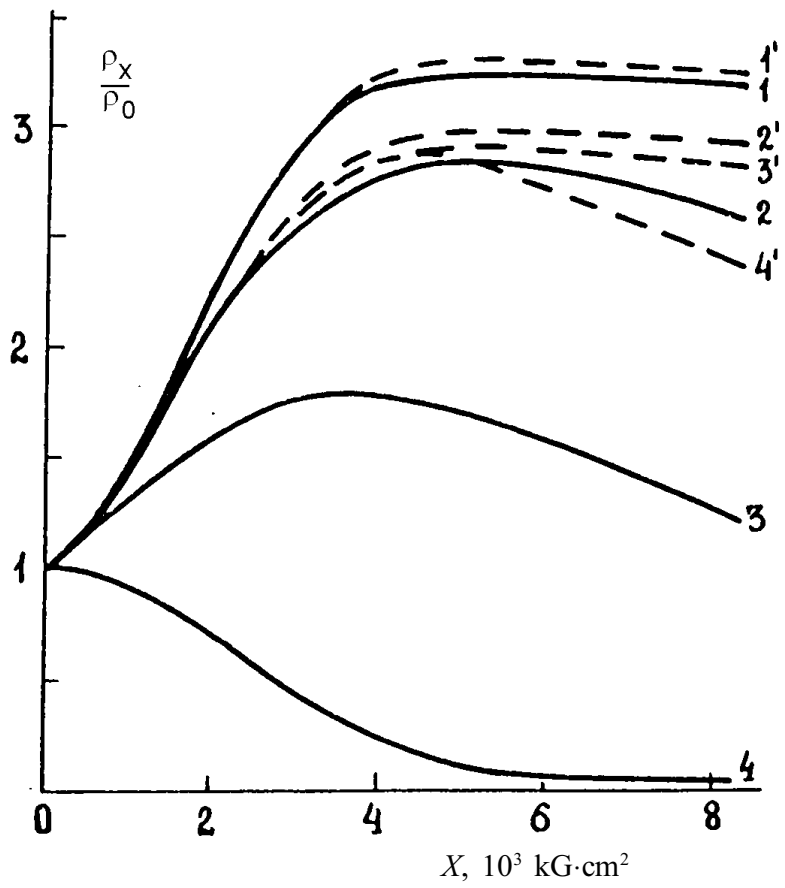

Fig. 1. Anisotropy of longitudinal tensoresistive effect $\rho_{x} / \rho_{0}=$ $=f(X)$ for equivalent crystallographic orientations of samples at $\mathrm{T}=78 \mathrm{~K}$ using different doses of $\gamma$-irradiation, $\Phi, 10^{17}$ quan$\mathrm{ta} / \mathrm{cm}^{2}: 1,1^{\prime}-0 ; 2,2^{\prime}-1.36 ; 3,3^{\prime}-1.76 ; 4,4^{\prime}-2.08$.

the $\rho_{X} / \rho_{0}=f(X)$ dependencies testify that at certain $\gamma$-exposure doses the mechanism of the redistribution of the charge carriers between $\Delta_{1}$ valleys of conduction band, which lead to the increase of crystal resistance with pressure $X \in[100]$ increase, is accompanied by the mechanism of the deformation-induced ionization of the radiation defect energy level $(A$-center, to which in the forbidden gap the acceptor level $E_{c}-0.17 \mathrm{eV}$ [ 15,24,25] corresponds). This leads to the increase of the current carriers concentration at the cost of decreasing energy difference between bottom of the conduction band and the level $E_{c}$ $-0.17 \mathrm{eV}$ with the pressure and respective resistance decrease. Because of the phosphor impurity donor concentration is essentially changed through the growth layers at time of $\gamma$-exposing, the resistance value and its dependence on the pressure for the samples of $\mathbf{I}$ group will be determined by the layers with smaller phosphor impurity concentration, that is by the parts with the bigger compensation degree (in series connection of the growth layers resistance). In samples of the second group, on the contrary, due to the parallel connection of the growth layers resistance, the resistance of the layers and its dependence from the pressure will be determined by the properties of the layers with the lesser degree of compensation. Presented considerations on the possible origin 


\section{Yu. P. Dotsenko al.: Electro-physical properties of $\boldsymbol{\gamma}$-exposed crystals ...}

of the tensoresistive effect anisotropy in $\gamma$-exposed silicon doped by phosphor during growth time are also confirmed in sufficient degree by the dependencies of the current carriers mobility on the exposure dose and by temperature dependencies of the resistance for the samples of I and II groups (Fig. 2).

Thus, the analysis of the presented results of the tensoresistive effect investigations for silicon doped during growth process by phosphor are testifying about the essential growth of the inessential for the initial samples gradients of the specific resistance at certain doses of $\gamma$ radiation due to different degree of donor impurity compensation by radiation defect ( $A$-center) with the acceptor properties.

More perfect method of semiconductor doping is, as is known, the doping by exposure of thermal (slow) neutrons [26]. In silicon, in particular, at exposure by thermal neutrons atoms of ${ }^{30} \mathrm{Si}$ isotope are transformed due to nuclear reaction into ${ }^{31} \mathrm{P}$ phosphor atoms $\left({ }^{30} \mathrm{Si}(n, \gamma) \rightarrow\right.$ $\left.\rightarrow{ }^{31} \mathrm{Si} \rightarrow{ }^{31} \mathrm{P}+\beta\right)$. Because the atoms of ${ }^{30} \mathrm{Si}$ isotope, which comprise approximately $3 \%$ from the number of silicon isotopes, are uniformly distributed in the crystal volume, method of transmutation doping enables to obtain also the uniform distribution of phosphor impurity, which gives a possibility to produce the semiconductor devices with the improved parameters in comparison with the devices on the base of semiconductors doped during the growth process by adding the impurity into the melt. The electro-physical properties of the transmutation

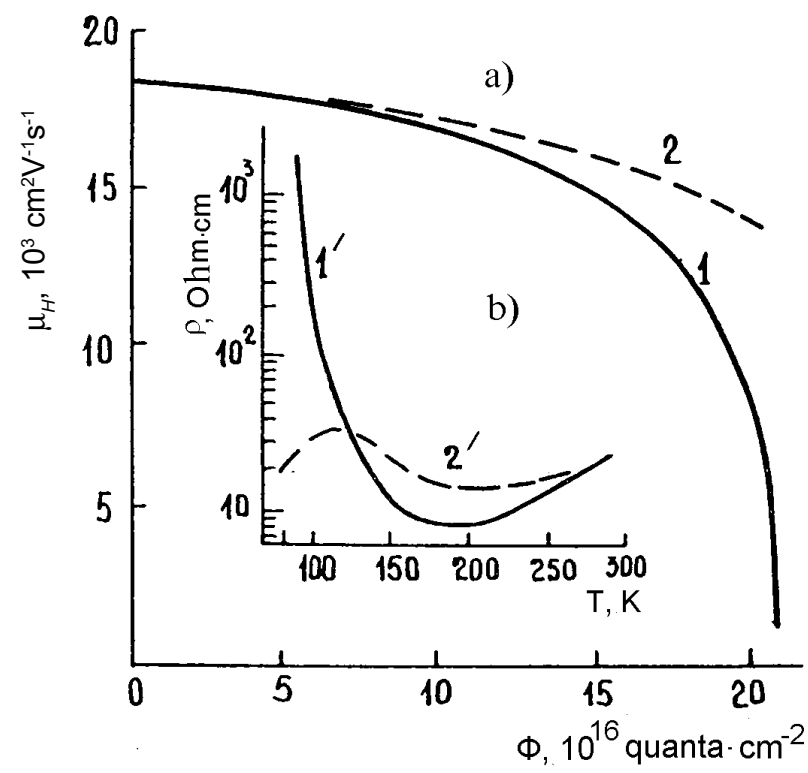

Fig. 2. Dependencies of the Hall mobility for current carriers (a) and resistivity (b) of $n$-Si (P) crystals $\left(N_{p}=1.2 \cdot 10^{14} \mathrm{~cm}^{-3}\right)$ for samples of the group I (curves 1 and 1', respectively) and samples of the group II (curves 2 and 2') on the dose of irradiation. doped silicon were studied in series of work [26-30].

The results of the tensoresistive effect investigation in crystals of neutron-doped silicon in the range of strong directed deformations [29-30] testify about the noticeable difference in the type of $\rho_{X} / \rho_{0}=f(X)$ dependencies recorded for the usually doped by phosphor silicon crystals and neutron-doped silicon at small values of impurity concentration $\left(N_{p} \leq 1.0 \cdot 10^{14} \mathrm{~cm}^{-3}\right)$. For silicon doped by phosphor during growth process, the $\rho_{X} / \rho_{0}=f(X)$ dependencies in the region of strong directed pressures have characteristic region of the tensoresistive effect saturation at $\mathrm{T}=78 \mathrm{~K}$ in pure crystals $\left(N_{p} \approx 1.0 \cdot 10^{12}\right.$ $1.0 \cdot 10^{14} \mathrm{~cm}^{-3}$ ) (Fig. 3, curve 1). The tensoresistive effect for the samples of the neutron doped silicon with comparable phosphor impurity concentration is characterized by the absence of saturation in the region of the strong directed pressures $\left(X>0.6 \cdot 10^{3} \mathrm{kG} \cdot \mathrm{cm}^{-2}\right.$, Fig. 3, curves 2,3$)$, which testify about the absence of complete ionization of phosphor impurity in such crystals at $\mathrm{T}=$ $=78 \mathrm{~K}$ and their additional ionization under the action of pressure in the $[100] \in \mathrm{X}$ direction. Thus, the Hall coefficient temperature dependence for the neutron doped n$\mathrm{Si}(\mathrm{P})$ in strain-free crystal $(X=0)$ has the noticeable decrease in the $78-130 \mathrm{~K}$ temperature region (inset to the Fig. 3, curve $2^{\prime}$ ). Similar interpretation is valid for the absense of saturation in the $\rho_{X} / \rho_{0}=f(X)$ dependencies, when considering the region of strong pressures for the doped during the growth process $n-\mathrm{Si}(\mathrm{P})$ which is compensated by the acceptor impurity (compensation degree

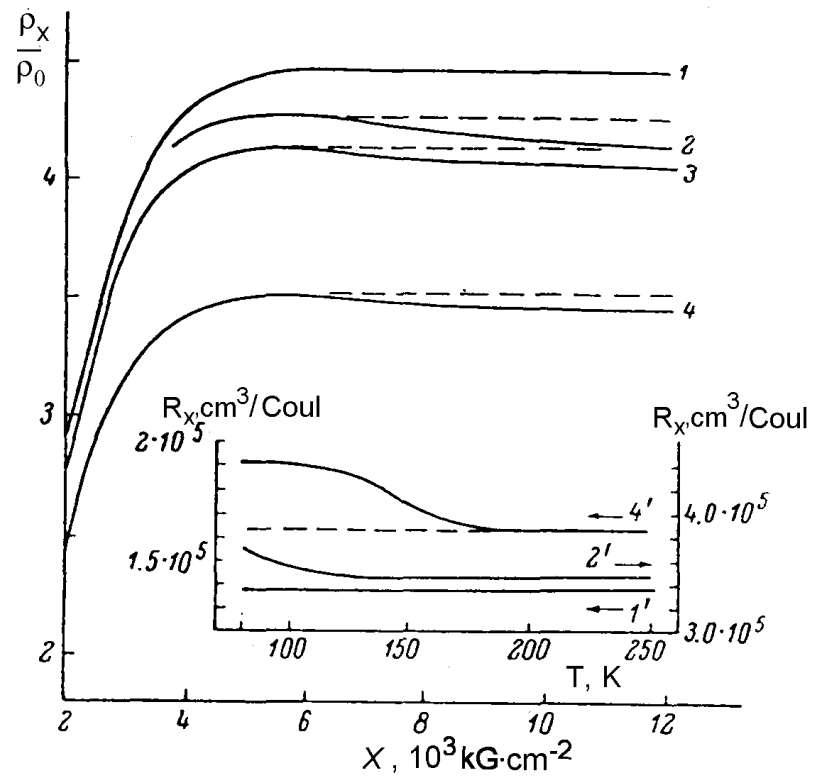

Fig. 3. Dependencies $\rho_{x} / \rho_{0}=f(X)$ obtained at $\mathrm{T}=78 \mathrm{~K}$ for $n-\mathrm{Si}$ samples doped by phosphorus in the course of growing (curves $1,4)$ and the transmutationally doped ones (curves 2,3 ). Temperature dependencies of the Hall coefficients for the samples $1,2,4$, are shown in the inset. 


\section{Yu. P. Dotsenko al.: Electro-physical properties of $\boldsymbol{\gamma}$-exposed crystals ...}

$k=N_{a} / N_{d} \approx 0,9$ ) (Fig. 3, curves $4,4^{\prime}$, respectively).

The deviation of the $\lg \left(n_{2} / n_{1}\right)=f(X)$ dependencies (where $\mathrm{n}_{2}$ - concentration of the electrons in valley, which is elevated in energy scale with the pressure, $n_{1}$ - concentration of the electrons in the descending valley) from linearity [31] (Fig. 4) also testify about the emergence of the additional to the inter-valley charge carrier redistribution mechanism of tensoresistive effect, determined by the increase of electron concentration, as in neutrondoped silicon crystals with phosphor concentration within $\approx(2.5 \div 20) \cdot 10^{13} \mathrm{~cm}^{-3}$, as well as in the compensated silicon. Therefore, it is apparent that for such crystals the method of the determination of the deformation potential constant from the slope of linear dependence $\lg \left(n_{2} / n_{1}\right)=F(X)$ [32] (in which the $n_{2} / n_{1}$ ratio of electron concentrations is expressed through the value of $\rho_{X} / \rho_{0}$ ratio, which is measured in the wide range of directed pressure changes, including the region of pressure saturation) cannot be used. The analysis of the main mechanisms of the tensoresistive effects, which lead to the deviation of the $\lg \left(n_{2} / n_{1}\right)=F(X)$ dependencies from linearity, is presented in thesis works [21,33].

As noted previously, transmutation doping of silicon enables to obtain the uniform distribution of the phosphor impurity, therefore with the aim to determine the influence of the $\gamma$-radiation on the properties of neutron-doped silicon the investigations of the physical properties of strongly-deformed [29] and $\gamma$-exposed with various doses crystals [30], were performed.

Neutron doping of the high resistance $p$-Si crystals which were grown in [100] direction was carried out in the reactor of the Institute of Nuclear Research as at NAS of Ukraine. After technological annealing which

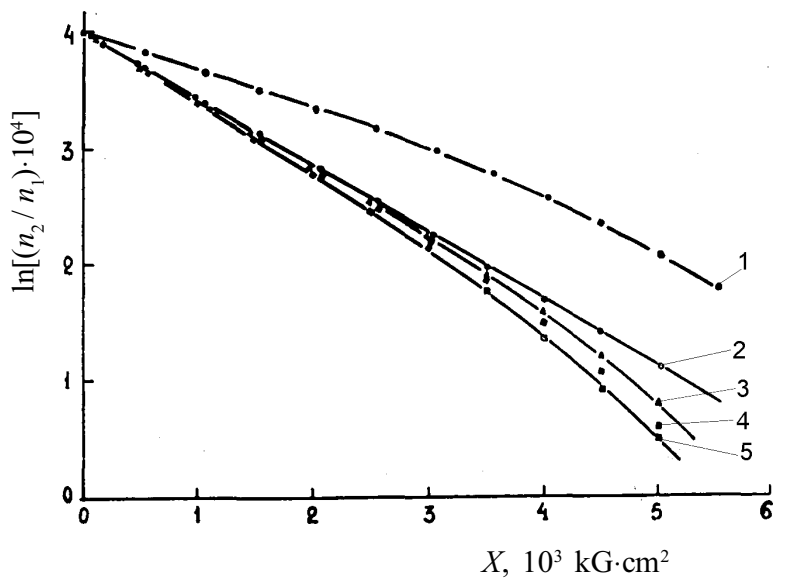

Fig. 4. Dependencies $\ln \left[\left(n_{2} / n_{1}\right) \cdot 10^{4}\right]=f(X)$ are plotted for $\mathrm{T}=$ $=150 \mathrm{~K}$ (curve 1 ) and $\mathrm{T}=78 \mathrm{~K}$ (curves $2-5$ ) for silicon crystals doped by phosphorus in the course of growing $(1,2,5)$ and neutron-doped $n$-Si (P) $(3,4)$ with parameters: $1,2-\rho_{300}=$ $=102 \mathrm{Ohm} \cdot \mathrm{cm}, k=N_{a} / N_{d} \approx 0.25 ; 3-\rho_{300}=220 \mathrm{Ohm} \cdot \mathrm{cm}, k \approx$ $\approx 0.25: 4-\rho_{300}=55 \mathrm{Ohm} \cdot \mathrm{cm}, k \approx 0.52 ; 5-\rho_{300}=103 \mathrm{Ohm} \cdot \mathrm{cm}$, $k \approx 0.9$. has been carried out with the aim of radiation damage removal, samples of $n$-Si had low phosphor concentration $N p=3.15 \cdot 10^{14} \mathrm{~cm}^{-3}$. Two groups of samples for measurements of longitudinal tensoresistive effects were oriented as in the work [22] in equivalent crystallographic directions [100] and [001]. Let us notice that the ratio of electrons concentrations, $n_{2} / n_{1}$, which determines the dependence of the specific resistance of silicon and germanium on pressure in the conditions of action of intervalley redistribution mechanism (Smith-Herring tensoeffects) can be respectively written for the non-degenerated crystals as:

$$
\frac{n_{2}}{n_{1}}=\left(1-\frac{\rho_{X}}{\rho_{s a t}}\right)\left(2 K \frac{\rho_{X}}{\rho_{s a t}}-2\right)^{-1}
$$

for $n$-Si in the conditions $\mathbf{X} \in \mathbf{J} \in[111]$.

$$
\frac{n_{2}}{n_{1}}=\left(\frac{\rho_{s a t}}{\rho_{X}}-1\right)\left(\frac{8 K+1}{3}-3 \frac{\rho_{s a t}}{\rho_{X}}\right)^{-1}
$$

for $n$-Ge in the conditions $\mathbf{X} \in \mathbf{J} \in[111]$.

Parameter $\mathbf{K}$ of mobility anisotropy in relations (1) and (2) is determined both for $n$-Si and $n$-Ge (for the above mentioned orientations ) by $\rho_{\text {sat }} / \rho_{0}$ ratio :

$K=\frac{3}{2} \frac{\rho_{\text {sat }}}{\rho_{0}}-\frac{1}{2}$

The results of the investigations of longitudinal tensoresistive effect (Fig. 5) testify about the absence of such high anisotropy of the obtained $\rho_{X} / \rho_{0}=f(X)$ dependencies even for the exposure doses of $\gamma$-quanta ${ }^{60} \mathrm{Co}$, which were essentially higher than those used in work [22]. The data obtained testify on the higher radiation stability in respect to the $\gamma$-exposure of neutron-doped silicon, which is necessary to take into account during the development of the solid state devices on the silicon base. The transmutation doping also provides, at respective control of slow neutron exposure dose, the possibility to obtain with the necessary accuracy the required concentration of the doping impurity with practical assurance of the creation of micro-nonuniformities, which are connected with the association creation: impurityimpurity or impurity - residual impurity atoms, which enables to exclude the creation most dangerous defects structures of single-crystal materials from the point of view of devices functioning reliability [29, 34, 35].

\section{Electrophysics properties of $\boldsymbol{\gamma}$ - exposed $\boldsymbol{n}$-germa- nium}

The results similar to the results obtained of tensoresistive effect anisotropy in $\gamma$-exposed $n$-Si were obtained by the authors of works $[19,36,37]$ under the investigations of the transport phenomena in the $\gamma$-exposed directed deformed $n$-Ge crystals. In the conditions of the crystal 


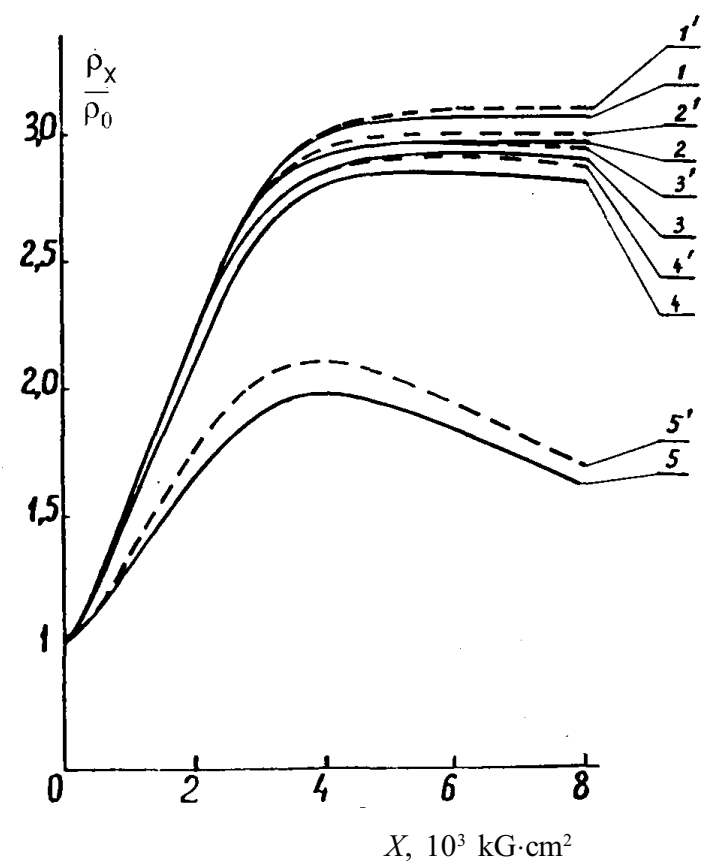

Fig. 5. Dependencies $\rho_{x} / \rho_{0}=f(X)$ for neutron-doped $n$-Si(P) $\left(N_{p}=3.15 \cdot 10^{14} \mathrm{~cm}^{-3}\right)$ obtained at different doses of $\gamma$-irradiation, $\Phi, 10^{17}$ quanta $/ \mathrm{cm}^{2}: 1,1^{\prime}-0 ; 2,2^{\prime}-1.36 ; 3,3^{\prime}-1.76 ; 4,4^{\prime}-$ $2.1 ; 5,5$ ' -8 . Solid and dash lines are obtained for samples orientated in equivalent crystallographic directions [001] (along the growth direction) and [100] (perpendicularly to the direction of crystal growth). The curves $1,1^{\prime}-4,4^{\prime}$ are obtained at $\mathrm{T}=78 \mathrm{~K}$, and the curves $5,5^{\prime}$ at $\mathrm{T}=300 \mathrm{~K}$.

growth in the [111] direction, the equivalent directions [111],[11ㅣ, [11 $\overline{1}]$ are placed at $110^{\circ}$ angle to the direction of the growth, which also enables to carry out the comparative experiments with the aim to detect the influence of $\gamma$-exposure on the anisotropy of tensoresistive properties of $n$-germanium, for which, as it is known, the maximal tensosensitivity is observed for the samples orientation along $\mathbf{X} \in\{111\} \in \mathbf{J}[38,39]$.

The results of the investigations of longitudinal tensoresistive effect in $\gamma$-exposed $n$-Ge crystals with the antimony impurity donor concentration $\approx 2.7 \cdot 10^{13} \mathrm{~cm}^{-3}[36]$. Presented in Fig. 6 results of measurements at $78 \mathrm{~K}$ testify about the increase of influence of the crystal defect structure, which is connected with non-uniform distribution of the doping impurity through the growing layers on the tensoresistive effect anisotropy, which was measured in equivalent directions $\{111\} \in \mathbf{X}$, with the increase of the $\gamma$-exposure dose (Fig. 6, curves 1, 1' - 4, $\left.4^{\prime}\right)$. Contrary to the experimental data for the $\gamma$-exposed $n$-Si(P), in $\gamma$-exposed $n-\mathrm{Ge}(\mathrm{Sb})$ crystals at $\mathrm{T}=78 \mathrm{~K}$ no additional ionization of charge carriers at pressures which reach values $1.2 \cdot 10^{3} \mathrm{kG} \cdot \mathrm{cm}^{-2}$ is observed. This data testify that under the directed deformation $\mathbf{X} \in$ [111] the

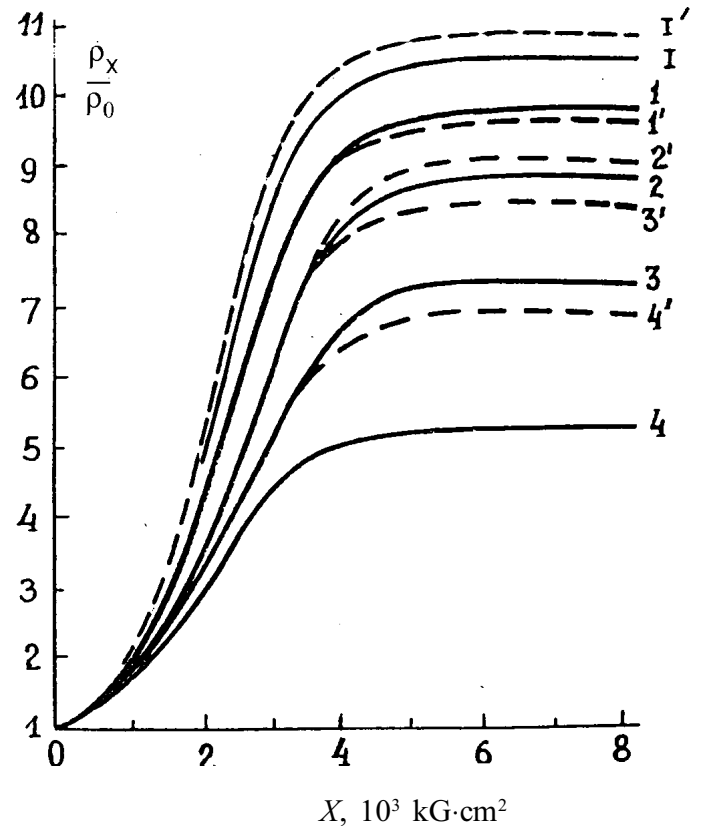

Fig. 6. Dependencies $\rho_{x} / \rho_{0}=f(X)$ are obtained for $n$-Ge(Sb) crystals at $\mathrm{T}=78 \mathrm{~K}$ and different doses of $\gamma$-irradiation, $\Phi$, $10^{16}$ quanta $/ \mathrm{cm}^{2}$ : I, I' and $1,1^{\prime}-0 ; 2,2^{\prime}-6.4 ; 3,3^{\prime}-8.8 ; 4,4^{\prime}-11$. Concentrations of doping Sb-impurity in Ge - crystals are as follows: I, I' $-N_{\mathrm{Sb}} \approx 1 \cdot 10^{12} \mathrm{~cm}^{-3} ; 1,1^{\prime}-4,4^{\prime}-N_{\mathrm{Sb}} \approx 2.7 \cdot 10^{13} \mathrm{~cm}^{-3}$.

shift of $E_{c}-0.2 \mathrm{eV}$ energy level (which belongs to the $\mathrm{V}_{2} \mathrm{D}$ defect of radiation origin [18]) in the direction towards the bottom of conductivity band $[11,37]$ is not sufficient for the additional ionization of the free charge carriers at $\mathrm{T}=78 \mathrm{~K}$.

Taking into account that the mobility anisotropy parameter $K=\mu_{\bar{B}} / \mu_{\|}$is determined for the inter-valley redistribution of electrons for the $n-G e(X \in J \in[111])$, as well as for the $n$-Si $(\mathbf{X} \in \mathbf{J} \in[100])$, by the relation (3), the essential decrease of the $\rho_{\mathrm{M}} / \rho_{0}$ value (Fig. 6, curves 1-4) cannot be connected, as it was noted earlier [21], with the increase of scattering anisotropy at single-charged radiation centers [31, 40], because at exposure dose $1.1 \cdot 10^{17}$ quanta $/ \mathrm{cm}^{2}$ their concentration is relatively low $\left(\sim 10^{13} \mathrm{~cm}^{-3}\right)$.

Let us note, that the compensated semiconductors are characterized by the presence of the current carriers scattering on the potential relief, amplitude $\sigma$ of which is determined as it was found in [41]:

$\sigma=\frac{e^{2} N_{t}^{2 / 3}}{\chi n_{s c r}^{1 / 3}}$

where $\chi$ is a dielectric constant, $n_{s c r}$ and $N_{t}$ are concentra- 


\section{Yu. P. Dotsenko al.: Electro-physical properties of $\boldsymbol{\gamma}$-exposed crystals ...}

tions of screening charge carriers and charged defects, respectively.

The change of the scattering mechanism in compensated crystals leads apparently to the significant decrease of the $\rho_{\mathrm{M}} / \rho_{0}$ value with the increase of the compensation degree also in $n$-Si crystals (Fig. 3, curves 1-4).

The data on the dose dependencies of the electrons mobility and $\rho_{\mathrm{M}} / \rho_{0}$ value of tensoresistive effect [21], which are presented in Fig. 7 also argues convincingly in favor of that. Not only the electron mobility, but also and the $\rho_{\mathrm{M}} / \rho_{0}$ value are essentially decreased for the samples in which the layers with the maximum compensation degree are included in series with the layers with minimal compensation degree (samples were prepared in the direction of crystal growth, see Fig. 7, curves 1,2). At the same time, the decrease of the electron mobility and $\rho_{\mathrm{M}} / \rho_{0}$ value for the samples with the parallel inclusion of layers with minimal and maximal compensation degrees (samples were prepared in the direction perpendicular to the axis of crystal growth) is essentially smaller (Fig. 7, curves $\left.1^{\prime}, 2^{\prime}\right)$. Let us notice, that tensoresistive effect, which is measured in $n$ - $\mathrm{Si}$ and $n$-Ge crystals in the equivalent directions ( $\{100\}-$ for $n$-Si and $\{111\}$ for $n-\mathrm{Ge}$ ) has small anisotropy also in unexposed samples, curves 1, 1' in Fig. 1, 5, 6 and curves I, I' in Fig. 6 were obtained by us for highly pure samples of $n$-Ge with insignificant doping level of antimony impurity $\left(\approx 10^{12} \mathrm{~cm}^{-3}\right)$.

We associate the presence of this difference in $\rho_{\mathrm{M}} / \rho_{0}$ value obtained in the region of tensoresistive effect satu-

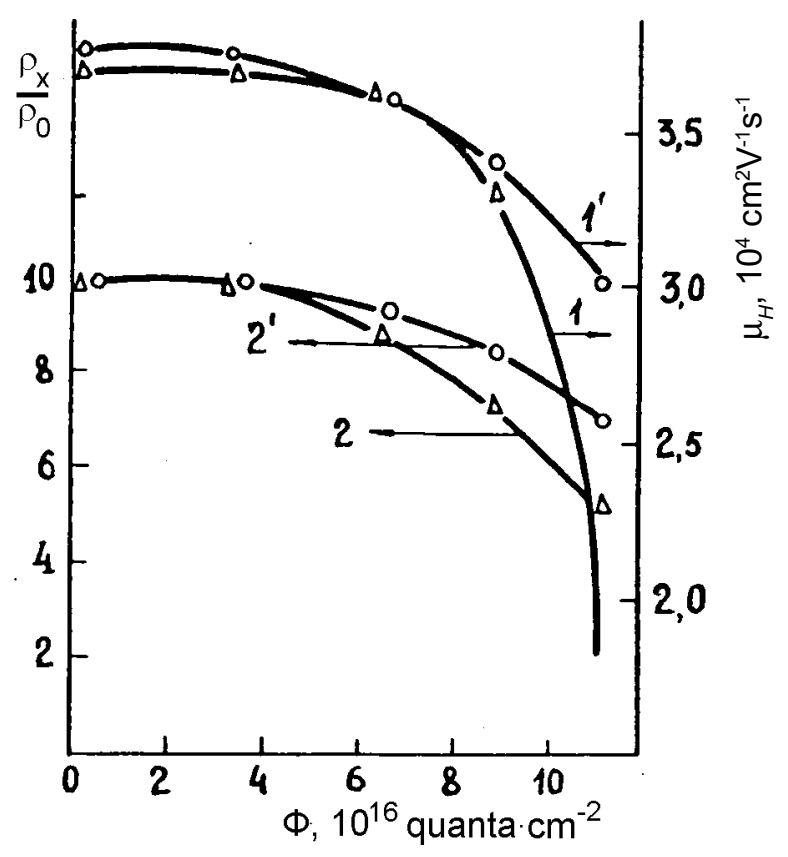

Fig. 7. Dose dependencies of the Hall mobility values for current carriers and for $\rho_{\infty} / \rho_{0}$ ratio in $n$-Ge crystals with the impurity concentration $N_{\mathrm{Sb}} \approx 2.7 \cdot 10^{13} \mathrm{~cm}^{-3}$ obtained at $\mathrm{T}=78 \mathrm{~K}$ for samples of the group I (curves 1,2) and the group II (curves 1', $\left.2^{\prime}\right)$, respectively. ration with residual deformations in crystals caused by the defect structure in the form of growth layers.

\section{Characteristics of the energy levels for the defects of radiation origin in $\gamma$-exposed $\mathrm{Si}$ and Ge crystals}

Due to the radiation influence the defects of structure originate in semiconductors, which create the series of complexes to which correspond different energy levels. Because the electrophysics properties of materials are directly connected with the characteristics of energy levels, identification of the level type and determination of the model for the respective radiation center are devoted by a number of works [15,18, 20, 24, 25, 40, 42-59]. On the base of analysis of electrophysics properties investigations of $\gamma$-exposed $\mathrm{Si}$ and Ge crystals it was established that in silicon, due to the creation of radiation centers (so called $A$-centers), the deep energy level $E_{c}-0.17 \mathrm{eV}$ arises in the forbidden gap $[15,24]$.

The model of $A$-center in silicon was proposed by authors [15] with taking into account the data on paramagnetic resonance investigations in the directly deformed crystals. It is determined that due to the $\gamma$-exposure the association of the vacancy with the oxygen atom arises, which under that is shifted from the lattice site in the direction of (100) plane. As follows from the results of theoretical calculations [42], an oxygen atom is stabilized in the site displaced along [100] direction. Closer shifted to it are also enclosing atoms of silicon, whereupon the crystal lattice in the place of $A$-center localization must have both hydrostatic and directed deformation component. It is established hereby, that $A$-center is characterized by the determined in the space axis (defect axis) which coincides with the crystallographic orientation [100] and belongs to such non-cubic centers the symmetry of which is lower than the crystal point symmetry and is determined by the point group $C_{2 V}$.

The results of the tensoresistive effect investigation also testify that the rate of the energy shift of the level, which belongs to $A$-center, is maximal when the deformation coincides with [100] direction.

Values of coefficient for the change of an energy gap between the silicon conoluetive band bottom and the $A$ center energy level were estimated for the different orientations of the deformation axis. Thus, for $\mathbf{X} \in$ [100] the value of such coefficient is $\alpha=3.9 \times 10^{-6} \mathrm{eV} \mathrm{cm} / \mathrm{kG}$; at the same time for the $\mathbf{X} \in$ [111] and $\mathbf{X} \in$ [110] this coefficient $\alpha \approx 1.4 \times 10^{-6} \mathrm{eV} \mathrm{cm} / \mathrm{kG}[21]$.

The values of the respective parameters, obtained in the work [30] on the base of analysis of the electrophysics properties of the $\gamma$-exposed neutron-doped $n$-Si(P) crystals, are: the ionization energy of the $A$-center $-E_{c}$ $0.14 \mathrm{eV}$, and the energy level change with the pressure for the $\mathbf{X} \in$ [100] orientation is characterized by the coefficient $\alpha=4.5 \cdot 10^{-6} \mathrm{eV} \mathrm{cm} / \mathrm{kG}$. Obtained were the parameters of the mobility anisotropy $\mathbf{K}$ and scattering anisotropy $\mathbf{K} \tau$ as were as values of deformation potential constant $\Xi_{\mathrm{u}}$ which were the same as for the $n$-Si doped 


\section{Yu. P. Dotsenko al.: Electro-physical properties of $\boldsymbol{\gamma}$-exposed crystals ...}

by phosphor during the course of growth. These results testify that the values of the elastic constant $C_{11}$ and $C_{12}$ for the silicon crystals doped by phosphor by different methods also coincide within the experimental accuracy limit values.

The essential anisotropy of the shift with pressure for the energy level $E_{c}-0.2 \mathrm{eV}$ of radiation center (complex: donor-divacancy $[18,40])$ was also observed in $\gamma$ exposed $n$-Ge crystals. Thus for the directions of the uniaxial pressure $\mathbf{X} \in$ [111] and $\mathbf{X} \in$ [110] the energy level $E_{c}-0.2 \mathrm{eV}$ shifts down relative to its position at $\mathrm{X}=0$, respectively, with the coefficients $5.6 \cdot 10^{-6} \mathrm{eV} \mathrm{cm} \mathrm{cm}^{2} / \mathrm{kG}$ and $1 \times 10^{-6} \mathrm{eV} \mathrm{cm} / \mathrm{kG}$, while for the orientation $\mathbf{X} \in[100]$ this level shifts upwards in accord to the factor $4 \cdot 10^{-6} \mathrm{eV} \mathrm{cm} / \mathrm{kG}[19]$.

Creation and annealing of the radiation defects in the grown by Chochralsky method and doped by germanium neutral impurity $p$-Si(B), with the oxygen concentration $\approx 8 \cdot 10^{17} \mathrm{~cm}^{-3}$ and the carbonone $\approx 5 \cdot 10^{17} \mathrm{~cm}^{-3}$, were investigated in the work [46] at doses of crystals $\gamma$ exposure which reach $5 \cdot 10^{17}-5 \cdot 10^{18}$ quanta $/ \mathrm{cm}^{2}$. Identified were two types of the radiation defects with the close values of energy levels $E_{v}+0.31 \mathrm{eV}$ and $\mathrm{E}_{\mathrm{v}}+0.35 \mathrm{eV}$, which previously have been associated with one type of defect, namely, the so called $K$-center. It is established that the correlation between concentrations of radiation defects which are created during the course of $\gamma$-exposure and to which the said energy levels correspond, depends on the presence of germanium impurity. It is also established that the rate of creation of the $\mathrm{C}_{\mathrm{O}} \mathrm{V}_{2}$ and $\mathrm{C}_{\mathrm{i}} \mathrm{O}_{\mathrm{i}}$ complexes depends on the state of oxygen and carbon impurities, which can be in isolated form as well as in the form of associations.

On the base of the analysis of the investigation results concerning the influence of the exposure and consequent annealing on the semiconductor properties, it was ascertained that the most active impurities which take part in the defects formation are oxygen and carbon. Stability of silicon devices are mainly determined be stability of radiation defects and connected with them deep energy levels of radiation origin, particularly, after the radiation treatment. It was determined, for example, that deep radiation centers which have carbon are not stable $[49,50]$.

In connection with the fact that both creation and structural transformation of radiation defects are directly associated with the initial defect structure of real crystals (background impurities, associations of background and doping impurities; enclosed by impurity atmosphere growth dislocations, etc.) the influence of background oxygen and carbon impurities on the above mentioned processes in silicon was studied [15-18, 24, 40, 46-54]; as well as doping (apart shallow impurities): Ge [46, 60], Pt $[55,56], \mathrm{Yb}[57]$; and doping by compensating impurities $[45,61]$, etc.

The properties of defects in $n$-silicon after exposure by $\gamma$ - quanta and particles which are created during the decomposition process of ${ }^{252} \mathrm{Cf}$ and consequent thermal annealing at temperatures up to $\approx 450{ }^{\circ} \mathrm{C}$ were investigated in the work [47]. It was found, that annealing at $\mathrm{T}=300{ }^{\circ} \mathrm{C}$ during 30 minutes leads to the creation of the defects ( $B$-centers), which are responsible for the activation energy of electrons transiting into conduction band. This energy equals to $\left(E_{c}-0.23\right) \mathrm{eV}$. It is assumed that $\mathrm{B}$-centers arise due to thermal activation of the defects of interstitial type as the concentration of B-centers is proportional to the exposure dose and does not depend on the type of exposure and doping level.

Transformation of the defects of vacancy type, which arises in the conditions of exposure by $\alpha$-particles at temperature $\mathrm{T}=77 \mathrm{~K}$ and annealed at temperature increase was studied in the work [60], when using the samples of single-crystal silicon grown by Chochralsky method and doped by phosphor and germanium impurities.

The parameters of the deep levels of radiation defects which arise during the process of the cyclic exposure of high-resistance silicon by $\alpha$-particles and its annealing at temperatures close to the room one and in the region $250-400{ }^{\circ} \mathrm{C}$ were determined in work [48]. Data obtained are important for predicting characteristics of detectors fabricated on the base of structures using high-resistance silicon, because the analysis of the results testify about the direct connection of the transformation reguliarities of radiation defects with the characteristics of the initial material and technology of structure fabrication. It is ascertained that the transformation of the radiation defects in $\mathrm{p}^{+}-\mathrm{n}-\mathrm{n}^{+}$structures from high-resistance silicon after cyclic exposure by $\alpha$-particles and consequent annealing is connected with the activation of the formation of complexes on the carbon base in crystals with the increased oxygen content.

Influence of $\gamma$-radiation on the properties of porous silicon was studied in works [62, 63].

It is necessary to notice that wide technological possibilities of ion implantation determine the intensive development of the reguliarities investigations of exposure influence by different elements ions on the formations and change of the defect structure of a subsurface semiconductor layer and its electrophysical properties.

\section{Conclusions}

Presented investigation data of the electrophysical properties of $\gamma$-exposed $n$-Si and $n$-Ge crystals testify about considerable increase of gradients in crystal resistivity with the increase of exposure dose. Increase of resistivity gradients is connected with the increase of compensation degree of donor impurity by radiation defects with acceptor properties. Thus, at significant exposure doses when the compensation degree approaches to unit, the crystal resistance increases, so that the measurements of electrophysical characteristics are noticeably complicated $[21,30]$, because the concentration of free charge carriers considerable decreases.

It is found also, that the neutron-doped $n$ - $\mathrm{Si}(\mathrm{P})$ crystals are more stable in relation to the $\gamma$-radiation in com- 


\section{Yu. P. Dotsenko al.: Electro-physical properties of $\boldsymbol{\gamma}$-exposed crystals ...}

parison with silicon doped by phosphor in the course of growing. Other characteristics of the mentioned materials, such as : constants of deformation potential, elastic constants, parameter of electron mobility anisotropy, coefficients which determine the shift rate of energy levels caused by radiation with pressure change for the deformation axis orientation along the main crystallographic directions, etc., within the limits of accuracy of the methods for their determination coincide.

Analysis of the tensoresistive effect investigations in strongly deformed $\gamma$-exposed crystals enables on the base of the determination of the shift rate of radiation defect energy level for different crystallographic directions of pressure to obtain certain conclusions about the microstructure of defect.

It is necessary to notice, that the defects of crystalline structure of semiconductors, which create deep energy levels in the forbidden gap (in particular, defects of radiation origin) determine, as useful consequences of their presence, impurity photoconductivity, high tenso- and thermal sensitivity, centers of high-speed recombination, etc., as weel as appearance of the undesirable consequences, namely: capture effects, oscillations, negative resistance, gradients increase, which are connected with the non-uniformities of the structure, etc.

Therefore, the investigations of the properties of semiconductors, determined by the peculiarities of the defect structure of actual crystals remain actual both from the scientific and the practical point of view, in particular, in the field of materials science, which investigates the change of physical properties under the influence of radiation.

\section{References}

1. P. R. Camp. Resistivity Striations in Germanium Crystals // J Appl. Phys. 25(4), pp.459-463. (1954).

2. H. Ueda. Resistivity Striations in Ge Single Crystals // J. Phys. Soc. Japan. 16(1), pp.61-66 (1961).

3. V. M. Turovskii, M. G. Milvinskii. Peculiarities of crystal growth from the melt by the Chokhralsky method // Fizika tverdogo tela 3 (9), pp. 2519-2524 (1961), in Russian.

4. H. C. Gatos et al. Impurity Striations in Unrotated Crystals of InSb // J. Appl. Phys. 32(10), pp. 2057 - 2058 (1961).

5. H. Frank. Lichtelectrisch Messung des Inneren electrischen Feld in inhomogenen Halbllitern // Czechoslov. Joum. Phys. 6(6), pp. 433-442 (1956).

6. P. I. Baranskii. The Peltier bulk effect in germanium// Zhournal tekhnicheskoi fiziki. 28(2), pp. 225-230 (1958), in Russian.

7. P. I. Baranskii, P. M. Kurilo. Dependence of the Peltier bulk effect on conductivity gradients // Fizika tverdogo tela 2(3), pp. 458462(1960), in Russian.

8. C. Herring. Effect of Random Ingomogenities on Electrical and Galvanomagnetic Measurements // J. Appl. Phys. 31(11), pp.1939 - 1964 (1960)

9. V. M. Babich, P. I. Baranskii, V. V. Gaiduchenko. Influence of nGe monocrystal layered structure on a magnetoresistance in strong magnetic fields // Fizika i tekhnika poluprovodnikov. 1(8), pp. 12711274 (1967), in Russian.

10. A. V. Fedosov, L. I. Panasyuk, Yu. Ya. Tkachuk. Influence of a layer growth on electrophysical properties of germanium and silicon. - In the book: Influence of defects and impurities on transport phenomena in silicon and germanium// The manuscript was deposited in Ukr. NIINTI, №2773, Uk., pp. 20-28 (1986), in Russian.

11. A. K. Semenyuk, A. V. Fedosov, P. F. Nazarchuk, V. R. Bukalo Piezoresistance of an irradiated n-Ge at presence of layered nonuniformities// Fizika i tekhnika poluprovodnikov. 16(7), pp. 12841287(1982), in Russian.

12. W. Spalek, H. Dorendorf. Widerstand-messungen an Feinstraifen in Germanium // Z. Angew. Phys. 29(6), pp. 344-346 (1970).

13. H. L. Frisch, J. A.Morrisson. High Field Magnetoresistance of Ingomogenous semiconductors and Plasmas - The stratified Medium // Ann. Phys. (USA). 26(2), pp. 181-221(1964).

14. V. M. Babich. Experimental examinations of the spectra and of the current carriers scattering anisotropy influence on galvanomagnetic effects in $\Pi-G e / /$ Abstr. of cand. thesis. Kiev, Inst. of Semicond., Academy of Sciences of Ukr SSR, 1969, 15p., in Russian.

15. G. D.Watkins, J. M. Corbett. Defects in Irradiated Silicon. ESR of the Si A-Center // Phys. Rev. - 121(4), pp. 1001-1014 (1961).

16. Radiation effects in semiconductors. Ed. L. S. Smirnov. Nauka, Novosibirsk (1979), 221p., in Russian.

17. V. L. Vinetskii, G. A. Kholodar. Radiation physics of semiconductors, Naukova dumka, Kiev (1979), 335p., in Russian.

18. V. V. Yemtsev, T. V. Mashovets. Impurities and point defects in semiconductors, Radio i svyaz, Moscow (1981), 248p., in Russian.

19. A. K. Semenyuk, A. V. Fedosov, P. F. Nazarchuk, Piezoresistance of n-Ge with radiation defects// Fizika i tekhnika poluprovodnikov, 14(9), pp. 1809-1811(1980), in Russian.

20. A. K. Semenyuk. Examination of radiation damages and their influence on kinetic effects in germanium and silicon // Abstr. of cand. thesis. Kiev, Inst. of Physics, Academy of sciences of Ukr SSR, 1969, 16p., in Russian.

21. A. V. Fedosov, Kinetic effects in multivalley semiconductors n-Si and $\mathrm{n}-\mathrm{Ge}$ in conditions of uniaxial elastic deformations // Doct. Thesis. Kiev, Inst. of Semicond., Academy of sciences of Ukr SSR, 1992, 315p., in Russian.

22. A. K. Semenyuk, A. V. Fedosov, L. I. Panasyuk, V. S. Timoshchuk. Piezoresistance of irradiated $\mathrm{n}$-Si with a layered distribution of impurity// Fizika i tekhnika poluprovodnikov. 20(3), pp. 545-547(1986), in Russian.

23. A. K. Semenyuk, A. V. Fedosov, L. I. Panasyuk, V. R. Bukalo, O. V. Kovalchuk. Peculiarities of layered non-uniformities influence on a piezoresistance in silicon single crystals // Izvestiya vuzov. Fizika, №1, pp. 115-116 (1989), in Russian.

24. I. D. Konozenko, A. K. Semenyuk, V. I. Khivrich. Radiation effects in silicon// Naukova dumka, Kiev (1974), 200p., in Russian.

25. A. I. Semenyuk, P. F. Nazarchuk. Influence of uniaxial deformation on an ionization energy of the A - centre in n-Si // Fizika $i$ tekhnika poluprovodnikov. 19(7), pp.1331-1333 (1985), in Russian.

26. Neutron transmutation doping of semiconductors. Ed. J. Miz. Mir, Moscow (1982). 264p., in Russian.

27. P. I. Baranskii, V. M. Babich, V. P. Borblik et al. Carrier current scattering mechanisms responsible for arising the magnetoresistance of $\mathrm{n}-\mathrm{Si}$ in the range of strong elastic deformations // Fizika $i$ tekhnika poluprovodnikov. 17(6), pp. 1064-1067(1983), in Russian.

28. P. I. Baranskii, V. V. Kolomoyets, A. V. Fedosov. Piezoresistance of usual and neutron-doped silicon crystals // Fizika i tekhnika poluprovodnikov. 5(5), pp. 864-867(1981), in Russian.

29. P. I. Baranskii, V. M. Babich, Yu. P. Dotsenko, V. V. Kolomoyets, V. P. Shapovalov. Influence of heat treatment on electrophysical properties of usual and neutron-doped silicon crystals // Fizika i tekhnika poluprovodnikov. 14(8), pp. 1546-1549 (1980), in Russian.

30. A. Ye. Gorin, N. N Dmytrenko, V. V. Kolomoyets, L. I. Panasyuk, A. V. Fedosov, V. I. Khivrich. Influence of strong directed deformation on properties of neutron-doped g-irradiatted silicon // Ukrainskii fizicheskii zhournal, 39(5), pp. 636-640 (1994), in Ukrainian.

31. P. I. Baranskii, I. S. Buda, I. V. Dakhovskii, V. V. Kolomoyets. Electrical and galvanomagnetic phenomena in anisotropic semi- 


\section{Yu. P. Dotsenko al.: Electro-physical properties of $\boldsymbol{\gamma}$-exposed crystals ...}

conductors, Naukova dumka, Kiev (1977), 269p., in Russian.

32. P. I. Baranskii, I. V. Dakhovskii, V. V. Kolomoyets, A. V. Fedosov. Determination of a deformation potential shear constant in silicon // Fizika i tekhnika poluprovodnikov. 10(7), pp.1387-1389. (1976), in Russian.

33. V. V. Kolomoyets. Physical principles of tensoeffects in multivalley semiconductors under extreme conditions// Doct. thesis. Kiev, Inst. of Semicond., Academy of Sciences of Ukr SSR, 1985, 348p., in Russian.

34. P. M. Henry, J. W. Farmer, J. M. Meess. Symmetry and electronic properties of the oxygen donor in pulled silicon // Appl. Phys. Lett. 45(4), pp.454-456 (1984).

35. Yu. P. Dotsenko, V. M. Ermakov, V. V. Kolomoets, V. F. Machulin, E. F. Venger, I. V. Prokopenko, N. M. Ponomarjev, B. A. Suss. Crystalline structure defects and strength of $\mathrm{Si}$ and $\mathrm{Ge} /$ / Inst. Phys.Conf. Ser. IOP Publishing Ltd., 1997-1998. №160, pp.281-284.

36. A. V. Fedosov, V. R. Bukalo, V. S. Timoshchuk. On anisotropy of a piezoresistance in irradiated $\mathrm{n}-\mathrm{Ge}$ with layered distribution of impurities // Fizika i tekhnika poluprovodnikov. 18(6), pp. 11351137 (1984), in Russian.

37. A. V. Fedosov, L. I. Panasyuk, V. S. Timoshchuk. Piezoresistance of the irradiated germanium// Fizika i tekhnika poluprovodnikov. 22(7), pp. 1297-1299(1988), in Russian.

38. G. L. Bir, G. Ye. Pikus. Symmetry and deformation effects in semiconductors, Nauka, Moscow (1972), 584p., in Russian.

39. V. V. Kolomoets. Influence of uniaxial elastic strain on resistance and magnitoresistance of $n-G e / /$ Doct. thesis. Kiev, Inst. of Semicond., Academy of Sciences of Ukr SSR, 1971, 178p., in Russian.

40. V. V. Yemtsev, T. V. Mashovets, E. A. Tropp. Kinetics of defects creation in semiconductors under series capture of several vacancies by an impurity atom// Fizika i tekhnika poluprovodnikov. 12(2), pp. 293-298 (1978), in Russian.

41. B. I. Shklovskii, A. L. Efros. Impurity band and conductance of compensated semiconductors // Zhournal eksperimentalnoi i teoreticheskoi fiziki. 60(2), pp.867-878 (1971), in Russian.

42. S. T. Pantelides, W. A. Harrison, F. Yudarian. Theory of offcenter impurities in semiconductors // Phys. Rev. B. 34(8), pp.60386040 (1986)

43. R. I. Agarwall, A. R. Ramdas. Effect of Uniaxial Stress on the Excitation Spectra of Donors in Silicon // Phys Rev.B. 137(2A), pp.602-612 (1965).

44. A. A. Lebedev, N. A. Sultanov, B. Ekke. Influence of uniaxial stress on a non-stationary capacitance spectroscopy of deep levels in $\mathrm{Si}(\mathrm{Zn}) / /$ Fizika i tekhnika poluprovodnikov, 21(2), pp.321324 (1987), in Russian.

45. L. S. Berman, A. A. Lebedev. Capacitance spectroscopy of deep centres in semiconductors, Nauka, Leningrad (1981), 176p., in Russian

46. V. I. Kuznetsov, P. F Luganov, A. P. Salmanov, A. V. Tsikunov. Accumulation and annealing of radiation defects in $\mathrm{p}-\mathrm{Si}(\mathrm{Ge}) / /$ Fizika i tekhnika poluprovodnikov, 23(4), pp. 1492-1495(1989), in Russian.

47. P. V. Kuchinskii, V. M. Lomano, L. M. Shakhlevich. Peculiarities of arising and properties of radiation defects in n-silicon after an irradiation followed by annealing // Fizika i tekhnika polupro- vodnikov, 28(11), pp. 1928-1936 (1994), in Russian.

48. Ye. M. Verbitskaya, V. K. Yeriomin, A. M. Ivanov, Z. Li, B. Shmidt. Generation of radiation defects in high-resistance silicon during cyclic irradiation and annealing // Fizika i tekhnika poluprovodnikov. 31(2), pp., 235-240 (1997), in Russian.

49. M. T. Asom, J. L Benson, R. Saner, L. C. Kimerling // Appl. Phys. Lett., 51, p.256, (1987).

50. L. W. Song, X. D. Zhan, B. W. Benson, G. D.Watkins // Phys. Rev., B42, p.5765, (1990).

51. Z. Su, A. Husain, J. W. Farmer // J. Appl. Phys., 67, p.1903 (1990).

52 I. L. Kolokovski, P. E. Luganov, V. V. Lukjanitsa, V. V. Shusha. Phys. Stat. Sol. (a), 118, 65 (1990).

53. I. I. Kolokovskii, P. E. Luganov, V. V. Shusha // Phys. Stat. Sol. (a), 127, p.103 (1991).

54. I. L. Kolokovski, V. V. Lukjanitsa. Peculiarities of accumulation of vacancy and interstitial defects in dislocationless silicon with different oxygen amount // Fizika i tekhnika poluprovodnikov, 31(4), pp.405-409 (1997), in Russian.

55. M. S. Yunusov, M. Akhmadaliyev, S. S. Sabirov. Processes of creation and annealing of radiation defects in p-Si (P, Pt) // Fizika i tekhnika poluprovodnikov. 29(4), p. 725 (1995), in Russian.

56. M. S. Yunusov, M. Karimov, M. Alikulov, A. Akhmadaliyev, B. L. Oksengendler, S. S. Sabirov. On peculiarities of a defect creation in p-Si (B, Pt) // Fizika i tekhnika poluprovodnikov. 31(2), pp.722-726 (1997), in Russian.

57. F. M. Talipov. Influence of ytterbium on radiation defect creation in silicon irradiated at $\mathrm{T}=77 \mathrm{~K} / /$ Fizika $i$ tekhnika poluprovodnikov. 31(6), pp.728-732 (1997), in Russian.

58. A. A. Lebedev. Capacitance spectroscopy of severe levels at presence of current carriers exchange with both allowed bands // Fizika i tekhnika poluprovodnikov, 31(4), pp.437-440 (1997), in Russian.

59. Kh. A. Abdullin, B. N. Mukashev. Defects in p-Si irradiated at 77 K. Energy spectrum and annealing kinetics // Fizika i tekhnika poluprovodnikov. 28(10), pp. 1831-1839 (1994), in Russian.

60. Kh. A. Abdullin, B. N. Mukashev. Investigation of vacancy defects in monocrystal line silicon irradiated at $\mathrm{T}=77 \mathrm{~K} / /$ Fizika $i$ tekhnika poluprovodnikov, 29(2), pp. 335-345 (1995), in Russian.

61. M. K. Bagdyrkhanov, K. A. Azizov, A. A. Tursunov, K. Kh. Khaidarov. Influence of $\gamma$ - irradiation on electrical and photoelectrical properties of silicon compensated by manganese // Fizika i tekhnika poluprovodnikov. 17(6), pp. 973-976 (1983), in Russian.

62. Ye. V. Astrova, V. V. Yemtsev, A. A. Lebedev, D. I. Poloskin, A. D. Remenyuk, Yu. V. Rud, V. Ve. Khartsiyev. Degradation of porous silicon photoluminescence forced by ${ }^{60} \mathrm{Co} \gamma$ - irradiation // Fizika i tekhnika poluprovodnikov. 29(7), pp.1301-1307(1995), in Russian.

63. Ye. V. Astrov, R. F. Vitman, V. V. Yemtsev, A. A. Lebedev, D. I. Poloskin, A. D. Remenyuk, Yu. V. Rud. Influence of $\gamma$ - irradiation on properties of porous silicon // Fizika i tekhnika poluprovodnikov. 30(3), pp.507-510 (1996), in Russian. 